\title{
CRESCIMENTO INICIAL DE Tabebuia aurea SOB TRÊS INTENSIDADES LUMINOSAS
}

\author{
Ademir Kleber Morbeck de Oliveira'; Sônia Cristina Juliano Gualtieri'
}

(recebido em 05.06.2010 e aceito para publicação em 08.07.2011)

\section{RESUMO}

A espécie Tabebuia aurea é comumente encontrada no Pantanal de Miranda, Mato Grosso do Sul, em formações vegetacionais conhecidas como paratudais. Essa espécie é considerada uma planta medicinal e sua madeira tem valor econômico, sendo utilizada na construção civil, entre outros usos, além de empregada na arborização e paisagismo. Levando-se em consideração seu amplo aproveitamento, seu desenvolvimento inicial foi analisado durante um período de 120 dias após a emergência, com o objetivo de avaliar o crescimento inicial da espécie em substrato com adição de matéria orgânica sob três intensidades luminosas visando a produção de mudas mais vigorosas, com menor custo. As plântulas foram colocadas em sacos plásticos, regadas diariamente até aproximadamente 80\% da capacidade de campo, sendo divididas em três grupos: o primeiro lote foi colocado em casa de vegetação com $45 \%$ de sombreamento artificial, o segundo, colocado em casa de vegetação coberta com tela de nylon, com 30\% de sombreamento artificial e o terceiro mantido a pleno sol (100\% luminosidade). As coletas foram mensais para a obtenção dos parâmetros altura, área foliar, peso da matéria seca da raiz, parte área e biomassa total, com o delineamento experimental realizado em blocos ao acaso, em esquema fatorial de quatro repetições $x$ três intensidades luminosas $x$ seis coleta. Os resultados obtidos indicam que as plântulas desenvolvem-se melhor a pleno sol ou sombreamento de $30 \%$, atingindo, respectivamente, 30,7 e $32 \mathrm{~g}$ de matéria seca e área foliar de $1.629,8$ e 1757,5 cm${ }^{2}$ aos 120 dias de cultivo. Para todas as luminosidades nas quais a espécie foi cultivada, esta apresentou elevada plasticidade fenotípica para o índice de robustez, indicando adaptação aos ambientes de cultivo, embora se desenvolva melhor em maiores luminosidades.

Palavras-chave: Sombreamento artificial; Análise de crescimento; Paratudo.

\footnotetext{
1 Professor Dr., Universidade Anhanguera-Uniderp, Programa de Pós-Graduação em Meio Ambiente e Desenvolvimento Regional, Campo Grande-MS. Email: akmorbeck@hotmail.com

${ }^{2}$ Professora Dra., Universidade Federal de São Carlos, Programa de Pós-Graduação em Ecologia e Recursos Naturais, São Carlos-SP. Email: dscp@ power.ufscar.br
} 


\section{INITIAL GROWTH OF Tabebuia aurea UNDER THREE LIGHT INTENSITIES}

\section{ABSTRACT}

Tabebuia aurea is easily found in Pantanal (swampland) of Miranda, Mato Grosso do Sul, in vegetation formations known as paratudais. This species is considered a medicinal plant and its wood has economic value, being important for building, afforestation and landscaping, among other uses. Considering its wide use, its initial development was analyzed for a period of 120 days after emergence. The aim of this study was to evaluate initial growth of species in substrate with addition of organic matter under three light intensities, in order to produce more vigorous plants at a lower cost. Seedlings were placed in plastic bags, watered daily until approximately $80 \%$ of field capacity and divided into three groups: the first was kept in greenhouse under $45 \%$ shade, the second, in greenhouse covered with nylon fabric with $30 \%$ shade, and the third remained in full sun (100\% brightness). Samples were collected monthly to obtain the parameters of height, leaf area, dry weight of roots, aerial part and total biomass, with the experiment conducted in a randomized block design in factorial arrangement of four repetitions $x$ three light intensities $x$ six collection times. The results obtained indicate that this species grows better under maximum brightness or $30 \%$ shading, reaching 30.7 and $32 \mathrm{~g}$ of dry material and 629.8 and $1757.5 \mathrm{~cm}^{2}$ of leaf area $\left(\mathrm{cm}^{2}\right)$, respectively, after 120 days of cultivation. Tabebuia aurea presents high phenotypic plasticity in terms of the index of robustness parameter, in the three treatments. This indicates that the species adapts well to all cultivation environments, but grows better at higher light intensity.

Keywords: Light intensity; Growth analysis; Paratudo.

\section{INTRODUÇÃO}

A espécie Tabebuia aurea ocorre em comunidades complexas na Região Amazônica, Nordeste, Centro-Oeste e Sudeste, em diversas formações vegetacionais, sendo popularmente conhecida por paratudo, caraíba, ipê-do-cerrado, entre outros nomes regionais (ALMEIDA et al., 1998; LORENZI, 2002), além de ser encontrada no Pantanal de Miranda, Mato Grosso do Sul, em áreas conhecidas como paratudais, onde normalmente apenas esta espécie arbórea domina a paisagem (SOARES e OLIVEIRA, 2009). 
A espécie é boa melífera, fornece corante amarelo para tintura em comunidades rurais e também é considerada uma planta medicinal (ALMEIDA et al., 1998). De acordo com Pott e Pott (1994) é um "santo remédio" do pantaneiro, que a utiliza para problemas diversos. Sua madeira tem valor econômico e é utilizada para confecção de ferramentas, móveis e construção civil, entre outros usos, além de empregada na arborização e paisagismo de jardins, ruas e parques (ALMEIDA et al., 1998; LORENZI, 2002).

Apesar de seu potencial de utilização, existem poucos trabalhos referentes a seu crescimento inicial (SILVA e NOGUEIRA, 2003; CABRAL et al., 2004; PACHECO et al., 2008), necessitando, portanto, de mais estudos básicos e aplicados. Freqüentemente a análise de crescimento é utilizada para predizer o grau de tolerância das diferentes espécies as mudanças na quantidade de radiação recebida, valendo-se de vários parâmetros para avaliação das respostas das plântulas em relação à intensidade luminosa (BENICASA, 2003).

Assim, o desenvolvimento das plantas é o resultado de correlações internas que envolvem carboidratos, reguladores de crescimento, água e minerais, embora a fotossíntese seja considerada o processo fisiológico fundamental para o crescimento, pois fornece matéria prima para o desenvolvimento da espécie (KITAJIMA, 1994; LARCHER, 2004), existindo uma grande diversidade de respostas quanto ao crescimento relacionado à luminosidade.

O desenvolvimento das espécies em diferentes condições luminosas é relacionada à sua capacidade em modificar os seus padrões de desenvolvimento em resposta às condições de luz. Porém a natureza destas respostas varia entre as espécies, de acordo com sua capacidade de aclimatação e a quantidade de luz, podendo-se classificar as espécies em dois grupos, plantas de sol ou de sombra, com base nas respostas morfológicas e fisiológicas a diferentes intensidades luminosas (OLIVEIRA et al., 2005).

A dinâmica do regime luminoso impõe dificuldades para o estabelecimento de espécies florestais, exigindo estratégias de crescimento tipo $r$, de crescimento rápido (plantas pioneiras) ou tipo $\mathrm{K}$, de crescimento lento (plantas clímax), condicionando a sobrevivência no meio. Desta maneira, conhecer a melhor condição luminosa para a espécie pode propiciar o desenvolvimento de plântulas em menor espaço de tempo (OLIVEIRA et al., 2005).

Assim, levando-se em consideração a possibilidade de múltiplos usos que Tabebuia aurea possui, o objetivo deste trabalho foi avaliar o crescimento inicial da espécie em 
substrato com adição de matéria orgânica sob três intensidades luminosas visando à produção de mudas mais vigorosas, com menor custo.

\section{MATERIAL E MÉTODOS}

Os frutos de Tabebuia aurea (Manso) B. et $\mathrm{H}$. foram colhidos de 10 matrizes

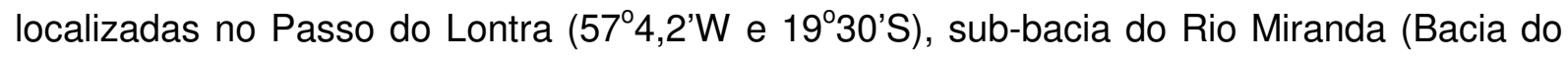
Alto Paraguai), no Pantanal de Miranda, Município de Corumbá, Mato Grosso do Sul.

Os frutos foram deixados ao sol durante 36 horas para deiscência natural e então, coletadas as sementes, separando-se manualmente aquelas em melhor estado de conservação. As mesmas foram acondicionadas em sacos de papel Kraft e transportadas para o Laboratório de Ecofisiologia Vegetal, Departamento de Botânica, Universidade Federal de São Carlos (UFSCar), São Paulo, onde foram colocadas para germinar em placas de Petri forradas internamente com uma folha de papel de filtro umedecido com solução do fungicida Captan $(0,2 \%)$ e mantidas sobre bancadas de laboratório sob temperatura ambiente, com umidade da placa verificada diariamente.

A porcentagem das sementes germinadas atingiu $90 \%$ no segundo dia e então, as plântulas foram colocadas em bandejas de alumínio contendo vermiculita, regadas diariamente com água destilada e, após uma semana, ao atingirem a altura de $7 \mathrm{~cm}$ acima do substrato, transplantadas para sacos de plantio (sacos plásticos pretos, com capacidade para $11 \mathrm{~kg}$, contendo substrato), sendo que cada saco plástico recebeu uma única plântula.

O substrato Latossolo Vermelho-Amarelo, unidade Canchim, de textura franco arenosa, foi coletado em área de Cerrado do Campus da UFSCar, a uma profundidade de 20 centímetros, peneirado em malha quadrada de $4,8 \mathrm{~mm}$ e, adicionado esterco bovino curtido (proporção de 2/1), homogeneizado e então enviado para análise. A região onde foi desenvolvido o experimento, cidade de São Carlos, apresenta clima classificado como Cwb, segundo Köeppen, com estações climáticas bem definidas, sendo uma seca, de abril a setembro e outra chuvosa, dezembro a fevereiro (região tropical). Segundo Nimer (1972), o clima é caracterizado como Tropical (pelo ritmo sazonal das precipitações), sub-quente (por apresentar temperatura média anual abaixo dos $22{ }^{\circ} \mathrm{C}$ e pelo menos um mês com temperatura média inferior a $18{ }^{\circ} \mathrm{C}$ ) e úmido (curta e pouco sensível estação seca no inverno), com médias anuais de precipitação entre 1200 e $1500 \mathrm{~mm}$.

O substrato apresentou as seguintes características: fósforo (Resina $\mu \mathrm{glcm}{ }^{3}$ ) -22 ; matéria orgânica (\%) - 3,6; $\mathrm{pH}\left(\mathrm{CaCl}_{2}\right)$ - 4,9; potássio $\left(\mathrm{emg} / 100 \mathrm{~cm}^{3}\right)$ - 0,6; cálcio $(\mathrm{emg} / 100$ 
$\mathrm{cm}^{3}$ ) - 1,2; magnésio $\left(\mathrm{emg} / 100 \mathrm{~cm}^{3}\right)$ - 1,1; alumínio $\left(\mathrm{emg} / 100 \mathrm{~cm}^{3}\right)-0,1$; soma das bases 2,9; volume (\%) - 52,7.

Os sacos plásticos contendo as plântulas, regadas diariamente pela manhã até aproximadamente $80 \%$ da capacidade de campo foram divididos em três grupos. O primeiro lote foi colocado em casa de vegetação com $45 \%$ de sombreamento artificial (radiação máxima obtida, $750 \mu \mathrm{mol} \mathrm{m} \mathrm{m}^{-2} \mathrm{~s}^{-1}$ ). $\mathrm{O}$ segundo grupo foi colocado em casa de vegetação coberta com tela de nylon conhecida comercialmente como "sombrite", com $30 \%$ de sombreamento artificial (radiação máxima obtida, $950 \mu \mathrm{mol} \mathrm{m} \mathrm{m}^{-2} \mathrm{~s}^{-1}$ ). $\mathrm{O}$ terceiro foi mantido a pleno sol (100\% luminosidade), sendo a radiação máxima obtida de $1200 \mu \mathrm{mol} \mathrm{m} \mathrm{m}^{-2}$. Os valores da radiação foram obtidos com o uso de um analisador portátil de gás infravermelho (ADC-LCA-2).

A partir de 37 dias pós plantio (DPP), feito em novembro, começaram a ser realizadas as coletas mensais (dezembro a maio) para a obtenção dos parâmetros altura, área foliar, peso da matéria seca da raiz, parte área e biomassa total, com o delineamento experimental realizado em blocos ao acaso, em esquema fatorial de quatro repetições $x$ três intensidades luminosas $x$ seis épocas de coleta, sendo utilizadas quatro plantas por tratamento, a cada coleta (quatro por tratamento, 12 por mês, 72 no total do experimento). Os dados foram analisados através do programa estatístico Bioestat 4.0 (AYRES et al., 2004) em nível de $5 \%$ de probabilidade e quando houve significância foi realizado o teste de média de Tukey, em nível de $5 \%(p<0,05)$.

Os parâmetros biométricos foram obtidos de acordo com as citações de Radford (1967) e Benicasa (2003), sendo altura (cm), área foliar $\left(\mathrm{cm}^{2}\right)$ e porcentagem de matéria orgânica investida em cada órgão (peso seco). O índice de plasticidade fenotípica (IPF) foi obtido pela equação (valor máximo do parâmetro avaliado - valor mínimo do parâmetro avaliado) / valor máximo do parâmetro avaliado, e índice de robustez (IR) $\left(\mathrm{g} \mathrm{cm}^{-1}\right)$, massa / comprimento, determinados de acordo com Valladares (2000).

Para determinação de peso seco, o material foi coletado, acondicionado em sacos de papel e então colocados em estufa de ventilação forçada a $80-90{ }^{\circ} \mathrm{C}$ durante $48 \mathrm{~h}$ e, depois de resfriados, pesados separadamente em balança analítica. 


\section{RESULTADOS E DISCUSSÃO}

A espécie apresentou capacidade de crescimento em todas as intensidades luminosas testadas. Porém os parâmetros avaliados indicaram crescimento diferenciado, em relação aos ambientes.

Em relação à altura, a espécie demonstrou tendência de crescimento contínuo até 120 dias pós plantio (DPP), sendo os resultados, na última coleta, estatisticamente iguais (F $=0.0075 ; \mathrm{gl} 2 ; \mathrm{p}=0.9933$ ) nas três intensidades luminosas, atingindo o máximo de 55,3, 55,3 e $55,1 \mathrm{~cm}$ de altura para 45 e $30 \%$ de sombreamento e a pleno sol, respectivamente, indicando que todas as condições luminosas foram adequadas para o crescimento em altura (Tabela 1).

TABELA 1 - Valores médios de altura $(\mathrm{cm})$ e área foliar $\left(\mathrm{cm}^{2}\right)$ de Tabebuia aurea após 30, 60, 90 e 120 dias, cultivadas em sacos plásticos em três condições luminosas.

TABLE 1 - Average values of height $(\mathrm{cm})$ and leaf area $\left(\mathrm{cm}^{2}\right)$ of Tabebuia aurea after 30, 60, 90 and 120 days cultivated in plastic bags in three light conditions.

\begin{tabular}{ccrrrrr}
\hline Dias & \multicolumn{2}{c}{$45 \%$ sombreamento } & \multicolumn{2}{c}{$30 \%$ sombreamento } & \multicolumn{2}{c}{ Pleno sol } \\
& Altura & Área Foliar & Altura & Área Foliar & Altura & Área Foliar \\
\hline 30 & $22,3 \mathrm{aA}$ & $138 \mathrm{aA}$ & $17,4 \mathrm{aB}$ & $105,1 \mathrm{aB}$ & $15,1 \mathrm{aB}$ & $102,2 \mathrm{aB}$ \\
60 & $34,3 \mathrm{bA}$ & $370 \mathrm{bA}$ & $30,4 \mathrm{bB}$ & $319,3 \mathrm{bB}$ & $28,4 \mathrm{bB}$ & $308,5 \mathrm{bB}$ \\
90 & $44,8 \mathrm{cA}$ & $802 \mathrm{cA}$ & $46,1 \mathrm{cA}$ & $649,7 \mathrm{cB}$ & $44,7 \mathrm{cA}$ & $639,3 \mathrm{Cb}$ \\
120 & $55,3 \mathrm{dA}$ & $1223 \mathrm{dA}$ & $55,3 \mathrm{dA}$ & $1630 \mathrm{~dB}$ & $55,1 \mathrm{dA}$ & $1758 \mathrm{Dc}$
\end{tabular}

* Médias seguidas pela mesma letra minúscula na coluna e maiúscula na linha não diferem entre, pelo teste de Tukey a $5 \%$ de probabilidade.

De acordo com Lutge (1997) e Larcher (2004), ocorre um ajuste das taxas metabólicas, com maior alocação de carbono para o caule quando a planta é submetida a condições de radiação que não são as apropriadas ao seu melhor crescimento, podendo modificar o diâmetro e altura da planta. Este fator foi observado, pois todos os tratamentos apresentaram resultados similares, demonstrando que provavelmente ocorreu uma adaptação da espécie, em relação à altura, com alocação de carbono para o sistema aéreo, o que permitiu um crescimento similar em todos os ambientes. Estes resultados mostram que a espécie, em relação a este parâmetro, possui uma grande capacidade adaptativa na parte inicial de seu desenvolvimento. 
Em relação ao parâmetro área foliar, há um aumento contínuo dos valores para todos os ambientes de cultivo; somente a partir de 90 DPP ocorrem diferenças significativas nos três ambientes, com indivíduos cultivados em $45 \%$ de sombreamento apresentando menor área, significativamente diferentes dos demais tratamentos, e indivíduos cultivados em $30 \%$ de sombreamento e a pleno sol, maiores valores, estatisticamente iguais ( $\mathrm{F}=$ 15.2805; gl 2; $p=0.0016$ ), com maior área obtida para plantas cultivadas em luminosidade máxima, aos 120 DPP $\left(1757,5 \mathrm{~cm}^{2}\right)$ (Tabela 1).

Levando-se em consideração que a alteração deste parâmetro é um mecanismo para compensar as baixas intensidades luminosas, de acordo com Larcher (2004) e Taiz e Zeiger (2009), a menor área foliar a partir de 120 DPP, em plantas cultivadas em 45\% de sombreamento indica a falta de adaptação da espécie, em relação à área foliar, para ambientes com maior nível de sombreamento. Desta maneira, em relação a este parâmetro, a espécie provavelmente apresenta um padrão de crescimento heliófilo a partir de 90 DPP, característico de espécies pioneiras ou secundárias iniciais, que necessitam de uma maior luminosidade para seu melhor desenvolvimento, dentro dos grupos sucessionais relacionados à vegetação, de plantas pioneiras, secundárias iniciais e tardias e clímaces.

Quanto ao peso seco, os maiores valores de peso seco da parte aérea foram obtidos quando as plantas foram cultivadas em maiores intensidades luminosas e, à medida que aumentou o tempo de cultivo, ocorreu uma maior diferença no crescimento, em relação ao ambiente que havia menor intensidade luminosa (Tabela 2).

TABELA 2 - Valores médios de peso seco (g) da parte aérea (PSPA), raiz (PSR) e total (PST) de Tabebuia aurea após 30, 60, 90 e 120 dias, cultivadas em sacos plásticos em três condições luminosas.

TABLE 2 - Average values of dry weight in grams of the aerial part (PSPA), root (PSR) and total (PST) of Tabebuia aurea after 30, 60, 90 and 120 days, cultivated in plastic bags in three light conditions.

\begin{tabular}{cllllllllll}
\hline Dias & \multicolumn{2}{c}{$45 \%$ sombreamento } & \multicolumn{3}{c}{$30 \%$ sombreamento } & \multicolumn{3}{c}{ Pleno sol } \\
& PSPA & PSR & PST & PSPA & PSR & PST & PSPA & PSR & PST \\
\hline 30 & $1 \mathrm{aA}$ & $0 \mathrm{aA}$ & $1 \mathrm{aA}$ & $1 \mathrm{aAB}$ & $0 \mathrm{aAB}$ & $1 \mathrm{aA}$ & $1 \mathrm{aB}$ & $0 \mathrm{aB}$ & $1 \mathrm{aA}$ \\
60 & $3 \mathrm{bAB}$ & $2 \mathrm{bA}$ & $4 \mathrm{bA}$ & $2 \mathrm{bA}$ & $1 \mathrm{bA}$ & $4 \mathrm{bA}$ & $3 \mathrm{bB}$ & $2 \mathrm{bA}$ & $5 \mathrm{bA}$ \\
90 & $5 \mathrm{cA}$ & $4 \mathrm{cA}$ & $9 \mathrm{cA}$ & $4 \mathrm{cB}$ & $5 \mathrm{cB}$ & $9 \mathrm{cAB}$ & $5 \mathrm{bBA}$ & $6 \mathrm{cC}$ & $10 \mathrm{cB}$ \\
120 & $9 \mathrm{dA}$ & $7 \mathrm{dA}$ & $16 \mathrm{dA}$ & $16 \mathrm{~dB}$ & $16 \mathrm{~dB}$ & $31 \mathrm{~dB}$ & $15 \mathrm{cB}$ & $17 \mathrm{~dB}$ & $32 \mathrm{~dB}$ \\
\hline
\end{tabular}


* Médias seguidas pela mesma letra minúscula na coluna e maiúscula na linha não diferem entre, pelo teste de Tukey a $5 \%$ de probabilidade.

O peso seco da parte aérea, nos três ambientes com intensidades luminosas distintas, é similar até 90 DPP. Aos 120 DPP, as plantas cultivadas sob $45 \%$ de sombreamento apresentam menores valores de acúmulo de biomassa, significantemente diferente dos demais, e as plantas crescidas sob $30 \%$ de sombreamento e a pleno sol se destacam, com os maiores valores, estatisticamente iguais ( $F=8.9930 ; g l 2 ; p=0.0074)$, sendo de 15,9 g (30\% de sombreamento) e 15,3 g (pleno sol). Todos os tratamentos apresentaram um aumento gradual no peso seco da parte aérea, com o decorrer do período (Tabela 2).

A estratégia de escape ao sombreamento com uma maior alocação de carbono para o caule quando a planta é submetida a condições de radiação inadequadas ao seu melhor crescimento é comum (LARCHER, 2004; TAIZ e ZEIGER, 2009); porém, para esta espécie, ao que parece, os ambientes mais sombreados produziram menor taxa de crescimento, indicando também falta de adaptação a estes ambientes, neste parâmetro, ou seja, um comportamento de espécie heliófila (pioneira ou secundária inicial). De acordo com alguns autores, como Pott e Pott (1994), a espécie é considerada pioneira no Pantanal, confirmando os dados obtidos. Novamente este comportamento fica mais evidente a partir de 90 DPP, também demonstrando que nos primeiros meses de crescimento, seu comportamento apresenta uma plasticidade maior.

O peso seco da raiz indica um aumento constante para a biomassa acumulada no sistema radicular, sendo que até 60 DPP as plantas apresentaram valores estatisticamente iguais ( $F=2.7457 ; g l=2 ; p=0.1165)$. A partir de $90 \mathrm{DPP}$, indivíduos cultivados sob $45 \%$ de sombreamento, menor valor e indivíduos cultivados a $30 \%$ de sombreamento e a pleno sol, maiores valores médios, estatisticamente iguais e significantemente diferentes de $45 \%$ de sombreamento ( $F=34.0228 ; \mathrm{gl} 2 ; \mathrm{p}<0.0001)$, chegando a $14,8 \mathrm{~g}(30 \%)$ e $16,8 \mathrm{~g}$ (pleno sol) aos 120 DPP (Tabela 2).

Larcher (2004) e Taiz e Zeiger (2009) afirmam haver uma hierarquia entre os locais que armazenam reservas e, que suprem os diferentes órgãos. Assim, a parte aérea de uma espécie pode ter uma maior prioridade na alocação de carbono, onde geralmente, os centros de crescimento são supridos com fotoassimilados provenientes da fonte mais próxima, sendo então a raiz suprida com carbono pelas folhas da base do caule. Em 
continuidade, as folhas centrais suprem o caule e as folhas do topo suprem as folhas jovens em crescimento.

Desta maneira, as folhas situadas na base do caule, que já sofrem um maior sombreamento causado pelas folhas situadas na parte superior do caule, podem ficar ainda mais prejudicadas pelo sombreamento artificial produzido nas casas de vegetação. Assim, quanto maior o sombreamento, provavelmente menor o peso seco da raiz e, quanto maior a intensidade luminosa, maior acúmulo de matéria seca. Este comportamento com maior acúmulo de matéria orgânica em ambientes menos sombreados, também indica que a espécie é mais adaptada para ambientes de maior intensidade luminosa, em situações encontradas no início do processo sucessional.

Em relação à biomassa total, ocorre um aumento significativo na biomassa seca, para indivíduos cultivados sob 30\% sombreamento e a pleno sol a partir de 120 DPP, quando plantas crescidas nestas intensidades luminosas apresentaram o maior acúmulo de biomassa (30,7 e $32 \mathrm{~g}$, respectivamente), sendo estatisticamente iguais e significativamente diferente de 45\% ( $F=137.8062 ; \mathrm{gl} 2 ; \mathrm{p}<0.00001)$, indicando que maior luminosidade propicia melhor crescimento (Tabela 2).

Em todos os tratamentos avaliados houve uma tendência constante de aumento de crescimento, porém, as plantas cultivadas com sombreamento de $45 \%$ apresentaram menor acúmulo de biomassa, indicando falta de adaptação, ou seja, uma espécie heliófila, pioneira ou secundária inicial. Também é observado que na fase inicial, até 90 DPP, o crescimento é similar, demonstrando plasticidade de comportamento. Após este período, a menor luminosidade é prejudicial, afetando negativamente o crescimento da espécie, resultados já observados para o parâmetro área foliar.

A proporção de assimilados alocados para a parte aérea e subterrânea sofreu oscilações no decorrer das coletas, o que pode observado no peso destes órgãos (Tabela 3). No sombreamento de $45 \%$ e $30 \%$, a parte aérea recebeu uma maior proporção de carbono durante todo o período de coleta. Já a pleno sol, até 60 DPP, o maior acúmulo foi para a parte aérea. Aos 90 e 120 DPP, o sistema subterrâneo acumulou uma maior proporção de matéria orgânica (Tabela 3). Estes dados demonstram que existe uma tendência constante de maior acúmulo no sistema radicular, porém mais acentuada no tratamento pleno sol (Tabela 3$)$, estatisticamente diferente dos demais tratamentos $(\mathrm{F}=$ $21.6441 ; g l 2 ; p=0.0006)$. 
TABELA 3 - Valores médios em gramas da relação MSPA/MSR de Tabebuia aurea após 30, 60, 90 e 120 dias cultivadas em sacos plásticos em três condições luminosas.

TABLE 3 - Average values in grams of relationship MSPA/MSR of Tabebuia aurea after 30, 60,90 and 120 days cultivated in plastic bags in three light conditions.

\begin{tabular}{lccc}
\hline Dias & $45 \%$ sombreamento & $30 \%$ sombreamento & Pleno sol \\
\hline 30 & $0,367 \mathrm{aA}$ & $0,571 \mathrm{aB}$ & $0,819 \mathrm{aC}$ \\
60 & $0,643 \mathrm{bA}$ & $0,619 \mathrm{bA}$ & $0,534 \mathrm{bA}$ \\
90 & $0,724 \mathrm{cA}$ & $1,027 \mathrm{cB}$ & $1,222 \mathrm{cC}$ \\
120 & $0,732 \mathrm{cA}$ & $0,928 \mathrm{cB}$ & $1,098 \mathrm{Cb}$ \\
\hline
\end{tabular}

* Médias seguidas pela mesma letra minúscula na coluna e maiúscula na linha não diferem entre, pelo teste de Tukey a $5 \%$ de probabilidade.

De acordo com Chazdon et al. (1996), respostas relacionadas à mudança na disponibilidade de luz podem ocorrer em nível foliar e/ou na planta toda, resultando em mudança no crescimento (alocação de biomassa), o que foi observado para a espécie $T$. aurea, onde as diferenças de luminosidade levaram a uma modificação na quantidade de biomassa alocada nos diferentes órgãos. Entretanto, os indivíduos podem responder de forma distinta aos diferentes níveis de intensidade luminosa. Uma modificação importante observada em plantas crescendo sob alta intensidade luminosa seria o maior investimento em biomassa do sistema radicular (POOTER, 1999), o que também foi observado para a espécie.

Um fator a ser considerado, de acordo com Medina (1982), é que também o baixo suprimento hídrico ou mineral leva a um aumento da alocação de carbono para a parte subterrânea em detrimento da parte aérea. A tendência desta espécie, ao que parece, é alocar quantidades significativas de carbono em suas raízes. Marschner (1995) chama este processo de "luxury uptake", que é comum quando existem altas concentrações externas de determinados macronutrientes; em condições de campo este processo ocorre durante a ontogenia da planta (ciclo de vida total), sendo um processo de grande importância, pois providencia uma reserva para períodos de alta demanda ou supressão do fluxo de nutrientes para a raiz.

A maior concentração de matéria orgânica no sistema radicular de $T$. aurea, além da questão da intensidade luminosa, também seria uma estratégia de sobrevivência durante os períodos desfavoráveis, pois uma possível alocação de fotoassimilados para a parte subterrânea propiciaria uma reserva, quando as condições ambientais forem adversas. 
Desta maneira, plantas crescidas sob maior intensidade luminosa, com maior reserva no seu sistema radicular, seriam as mais adaptadas para um possível estresse hídrico ou nutricional, o que, de acordo Soares e Oliveira (2009), são tipos de estresse comuns em áreas de Paratudal, no Pantanal de Miranda, local de coleta das sementes.

Em relação ao índice de robustez (Tabela 4), todas as plantas apresentaram igual vigor, com uma tendência de crescimento constante, com o maior valor encontrado, 0,05 g $\mathrm{cm}^{-2}$, aos 120 dias de experimento, para todas as intensidades luminosas, sendo estatisticamente iguais todos os tratamentos $(F=3.5970 ; \mathrm{gl} 2 ; \mathrm{p}=0.0704)$.

TABELA 4 - Valores médios em $\mathrm{g} \cdot \mathrm{cm}^{-2}$ de índice de robustez de Tabebuia aurea após 60, 90 e 120 dias cultivadas em sacos de plantio em três condições luminosas.

TABLE 4 - Average values in $\mathrm{g} \cdot \mathrm{cm}^{-2}$ of index of robustness of Tabebuia aurea after 60,90 and 120 days cultivated in planting bags in three light conditions.

\begin{tabular}{lrrr}
\hline Dias & $45 \%$ sombreamento & $30 \%$ sombreamento & Pleno sol \\
\hline 30 & $0,0045 \mathrm{aA}$ & $0,0040 \mathrm{aA}$ & $0,0059 \mathrm{aA}$ \\
60 & $0,0131 \mathrm{bA}$ & $0,0118 \mathrm{bA}$ & $0,0134 \mathrm{bA}$ \\
90 & $0,0196 \mathrm{cA}$ & $0,0200 \mathrm{cA}$ & $0,0210 \mathrm{cA}$ \\
120 & $0,0463 \mathrm{dA}$ & $0,0504 \mathrm{dA}$ & $0,0475 \mathrm{dA}$ \\
\hline
\end{tabular}

* Médias seguidas pela mesma letra minúscula na coluna e maiúscula na linha não diferem entre, pelo teste de Tukey a $5 \%$ de probabilidade.

Os resultados encontrados indicam que todos os tratamentos produziram plantas com a mesma robustez. Apesar das diferenças no acúmulo de biomassa e crescimento, os três ambientes produziram plantas robustas, aptas a ser estabelecer no ambiente. Este fator também indica que a espécie, apesar de crescimento melhor a pleno sol, também consegue crescer com vigor em ambientes sombreados, pelo menos no período avaliado, de 120 DPP. Os índices de plasticidade encontrados $(0,91 ; 0,92 ; 0,87)$ são estatisticamente iguais entre si $(F=0.2342 ; g l 2 ; p=0.7974)$ para as três condições luminosas, demonstrando que no parâmetro robustez, a espécie apresenta uma grande habilidade de alterar sua morfologia em decorrência de interações ambientais, embora plantas crescidas a pleno sol apresentem maior taxa de acúmulo de biomassa.

Levando-se em consideração os dados obtidos, a análise de crescimento em plantas apresenta-se como uma técnica importante para integrar informações morfo-fisiológicas e inferir prováveis respostas dos vegetais às mudanças ambientais. 


\section{CONCLUSÕES}

Tabebuia aurea apresenta maior acúmulo de biomassa e área foliar quando cultivada em maior intensidade luminosa. Para todas as luminosidades nas quais a espécie foi mantida, os indivíduos apresentaram elevada plasticidade fenotípica para o índice de robustez, indicando adaptação aos ambientes de cultivo, embora com menor acúmulo de biomassa para pouca luz.

A espécie se desenvolve melhor em ambientes com maior luminosidade, indicando comportamento de espécie pioneira ou secundária inicial, de acordo com classificação sucessional, acumulando, a partir de 90 DPP, a maior parte dos fotoassimilados na raiz.

\section{AGRADECIMENTOS}

Ao CNPq pela bolsa concedida aos dois autores, que também agradecem a contribuição dos consultores para a correção do artigo e a Universidade AnhangueraUniderp pelo financiamento do projeto, através da liberação de recursos (Grupo Interdisciplinar de Pesquisa-GIP).

\section{REFERÊNCIAS BIBLIOGRÁFICAS}

ALMEIDA, S.P.; PROENÇA, C.E.B.; SANO, S.M.; RIBEIRO, J.F. Cerrado: espécies vegetais úteis. Planaltina: EMBRAPA - CPAC, 1998. 464p.

AYRES, M.; AYRES Jr, M.; AYRES, D.L.; SANTOS, A.S. BioEstat 4.0: Aplicações estatísticas nas áreas das ciências biológicas e médicas. Manaus: Sociedade Civil Mamirauá, 2004. 364p.

BENICASA, M.M.P. Análise de crescimento de plantas (noções básicas). 2ed. Jaboticabal: FUNEP, 2003. 41p. 
CABRAL, E.L.; BARBOSA, D.C.A.; SIMABUKURO, E. A. Crescimento de plantas jovens de Tabebuia aurea (Manso) Benth. \& Hook. f. ex S. Moore submetidas a estresse hídrico. Acta Botanica Brasilica, São Paulo, v.18, n.2, p.241-251, 2004.

CHAZDON, R.L.; PEARCY, R.W.; LEE, D.W.; FETCHER, N. Photosynthetic response of tropical forest plants to contrasting light environments. In: MULKEY, S.S.; CHAZDON, R.L.; SMITH, A.P. (Eds). Tropical forest plant ecophysiology. New York: Chapman Hall, p.555. 1996.

KITAJIMA, K. Relative importance of photosynthetic traits and allocation patterns as correlates of seedling shade tolerance of 13 tropical trees. Oecologia, Heidelberg, v.98, n.3/4, p.419-428, 1994.

LARCHER, W. Ecofisiologia vegetal. São Carlos: RiMa, 2004. 531p.

LORENZI, H. Árvores brasileiras: manual de identificação e cultivo de plantas arbóreas nativas do Brasil. Vol. 1. 4 ed. Nova Odessa: Plantarum, 2002, 384p.

LUTGE, U. Physiological ecology of tropical plants. Berlim: Springer-Verlag, 1997. 289p.

MARSCHNER, H. Mineral nutrition of higher plants. London: Academic Press, 1995. $889 p$.

MEDINA, E. Physiological ecology of neotropical savanna plants. In: HUNTLEY, B.J.; WALTER, B.H. (Eds.) Ecology of tropical savannas. Berlin: Springer-Verlag, 1982, p.308335.

NIMER, E. Climatologia da região sudeste do Brasil. Revista Brasileira de Geografia, Rio de Janeiro, v. 34, n. 1, p. 3-38, 1972.

OLIVEIRA, A.K.M.; LAURA, V.A.; PEREZ, S.C.J.G.A. A influência da luminosidade no desenvolvimento vegetal. In: BAUER, F.C.; VARGAS JUNIOR, F.M. Produção e Gestão Agroindustrial. Campo Grande: Uniderp, 2005, p. 97-118. 
PACHECO, M.V.; MATOS, V.P.; FELICIANO, A.L.P.; FERREIRA, R.L.C. Germinação de sementes e crescimento inicial de plântulas de Tabebuia aurea (Silva Manso) Benth. \& Hook f. ex S. Moore. Revista Ciência Florestal, Santa Maria, v.18, n.2, p.143-150, 2008.

POOTER, L. Growth responses of 15 rains forest tree species to a light gradient: the relative importance of morphological and physiological traits. Functional Ecology, Oxford, v.13, n.3, p.396-410, 1999.

POTT, V.J.; POTT, A. Plantas do Pantanal. Corumbá: EMBRAPA-SPI, 1994. 320p.

RADFORD, P.J. Growth analysis formulae - their use and abuse. Crop Science, Madison, v.7, n.3, p.171-175, 1967.

SILVA, E.C.; NOGUEIRA, R.J.M.C. Crescimento de quatro espécies lenhosas cultivadas sob estresse hídrico em casa-de-vegetação. Revista Ceres, Viçosa, v.50, n.288, p.203-217, 2003.

SOARES, J.J.; OLIVEIRA, A.K.M. Os paratudais no Pantanal de Miranda. Revista Árvore, Viçosa, v.33, n.2, p.339-347, 2009.

TAIZ, L.; ZEIGER, E. Fisiologia vegetal. 4. ed. Porto Alegre: Artmed, 2009. 719p.

VALLADARES, F. Light and plant evolution: adaptation to the extremes versus phenotypic plasticity. In: GREPPIN, H. Advanced studies in plant biology. Geneve: University of Geneve, p.341-355. 2000. 